\title{
A solar surface dynamo
}

\author{
A. Vögler ${ }^{\star}$ and M. Schüssler \\ Max-Planck-Institut für Sonnensystemforschung, Max-Planck-Strasse 2, 37191 Katlenburg-Lindau, Germany \\ e-mail: [voegler;msch] @mps .mpg.de \\ Received 7 February 2007 / Accepted 21 February 2007

\section{ABSTRACT}

\begin{abstract}
Context. Observations indicate that the "quiet" solar photosphere outside active regions contains considerable amounts of magnetic energy and magnetic flux, with mixed polarity on small scales. The origin of this flux is unclear. observed magnetic flux.

Methods. We have carried out MHD simulations of solar surface convection, including the effects of strong stratification, compressibility, partial ionization, radiative transfer, as well as an open lower boundary. simulation run with a magnetic Reynolds number of about 2600. The magnetic energy approaches saturation at a level of a few percent of the total kinetic energy of the convective motions. Near the visible solar surface, the (unsigned) magnetic flux density reaches at least a value of about $25 \mathrm{G}$.

Conclusions. A realistic flow topology of stratified, compressible, non-helical surface convection without enforced recirculation is capable of turbulent local dynamo action near the solar surface.
\end{abstract} \\ Aims. We test whether local dynamo action of the near-surface convection (granulation) can generate a significant contribution to the \\ Results. Exponential growth of a weak magnetic seed field (with vanishing net flux through the computational box) is found in a
}

Key words. Sun: magnetic fields - Sun: photosphere - magnetohydrodynamics (MHD)

\section{Introduction}

Magnetic fields in the quiet solar photosphere (often referred to as "internetwork fields") are of considerable interest in connection with the heating of the upper solar atmosphere and as a possible example of local fast dynamo action. Owing to their apparently "turbulent" nature, with mixed polarity on small spatial scales, their extended range of field strengths, and their weak polarization signals, quiet-Sun (QS) magnetic fields are a difficult observational target. Quantitative results from observations based upon different methods do not yet provide a fully consistent picture (e.g., Lin \& Rimmele 1999; Sánchez Almeida \& Lites 2000; Khomenko et al. 2003; Sánchez Almeida et al. 2003; Domínguez Cerdeña et al. 2003; Lites \& Socas-Navarro 2004; Domínguez Cerdeña et al. 2006a,b; Trujillo Bueno et al. 2004; Sánchez Almeida 2003, 2005), but it appears to be established that small-scale, mixed-polarity magnetic fields are ubiquitous in the quiet Sun and contribute significantly to the total magnetic energy and unsigned flux in the photosphere outside active regions.

The origin of these small-scale fields is not yet fully clarified. It seems plausible that rise and emergence of magnetic flux from the deep convection zone in the form of small bipoles as well as the debris from decaying active regions contribute to the QS magnetic fields. On the other hand, the increasing flux replenishment rates towards smaller scales (Hagenaar et al. 2003) and the absence of a significant variation of the QS flux between solar minimum and maximum (Trujillo Bueno et al. 2004) indicate that a considerable part of the QS magnetic fields may be produced by a self-excited turbulent dynamo operating in the

^ Present address: Sterrekundig Instituut, Utrecht University, Postbus 80000,3508 TA Utrecht, The Netherlands. near-surface layers of the Sun (e.g., Petrovay \& Szakaly 1993). Since the turnover time of the corresponding dominant convection pattern (granulation) of about $10 \mathrm{~min}$ is much smaller than the solar rotation period, this flow is practically unaffected by the Coriolis force and thus exhibits no net helicity. The principle of such a non-helical surface dynamo has been demonstrated in incompressible closed-box MHD simulations of thermal convection at high Rayleigh number (Cattaneo 1999; Cattaneo et al. 2003). The question is whether these results carry over to the case of realistic solar granulation. This is not at all obvious since numerical simulations of solar surface convection show that the powerful surface cooling by radiation and the strong stratification prevent a significant recirculation of the downflowing plasma in the near-surface layers (Stein \& Nordlund 1989). As a consequence, small-scale magnetic flux is rapidly pumped into the deeper layers of the convection zone and is thus lost for further amplification by the near-surface flows (Stein \& Nordlund 2003). In a simulation with artificially closed boundaries, such pumping is suppressed and a strong local recirculation maintained, so that a local dynamo is efficiently sustained.

In this paper, we present the first example of dynamo action in a realistic simulation of solar surface convection with an open lower boundary. Our results indicate that a realistic flow topology of strongly stratified, compressible, and non-helical surface convection without enforced recirculation is capable of driving a turbulent local dynamo.

\section{Numerical model}

We have used the MURaM code (Vögler et al. 2005; Vögler 2003) to carry out local-box MHD simulations of solar surface convection with grey radiative transfer. The computational 
Table 1. Simulation parameters. The estimate of the magnetic Reynolds number is based on the rms flow velocity and a length scale of $1 \mathrm{Mm}$ (granulation scale). $\Delta z$ and $\Delta x$ are the vertical and horizontal grid spacing, respectively.

\begin{tabular}{ccccc}
\hline \hline Run & $\begin{array}{c}\text { Height/width } \\
{[\mathrm{Mm}]}\end{array}$ & $\begin{array}{c}\Delta z / \Delta x \\
{[\mathrm{~km}]}\end{array}$ & $\begin{array}{c}\eta \\
{\left[10^{10} \mathrm{~cm}^{2} \mathrm{~s}^{-1}\right]}\end{array}$ & $\begin{array}{c}R_{\mathrm{m}} \\
\text { (approx.) }\end{array}$ \\
\hline $\mathrm{A}$ & $1.4 / 6.0$ & $14 / 20.8$ & 11.1 & 300 \\
$\mathrm{~B}$ & $1.4 / 6.0$ & $14 / 10.4$ & 2.5 & 1300 \\
$\mathrm{C}$ & $1.4 / 4.86$ & $10 / 7.5$ & 1.25 & 2600 \\
\hline
\end{tabular}

domain covers the height range between about $800 \mathrm{~km}$ below and $600 \mathrm{~km}$ above the average height of the visible solar surface and has a horizontal extension between about $5 \mathrm{Mm}$ and $6 \mathrm{Mm}$ (see Table 1).

The side boundaries are periodic in both horizontal directions. The boundary condition at the bottom $(z=0)$ permits free in- and outflow of matter: the upflows are assumed to be vertical, $v_{x}=v_{y}=0, \partial_{z} v_{z}=0$, in the downflows the vertical gradients are set to zero, $\partial_{z} v_{x}=\partial_{z} v_{y}=\partial_{z} v_{z}=0$. The upper boundary is closed for the flow. The magnetic field is assumed to be vertical at the upper and lower boundaries: $B_{x}=B_{y}=0, \partial_{z} B_{z}=0$. Further details of the boundary conditions are described in Vögler et al. (2005). Horizontal fields which get carried towards the lower boundary in downflows nevertheless leave the simulation domain by means of magnetic diffusion across the boundary: at any thime, the diffusive boundary layer at the bottom adjusts its width such that the resulting diffusive flux matches the incoming advection flux into the boundary layer. Since the magnetic field strength in the lower part of the box always remains significantly below the equipartition value with the convective flows, it is thus guaranteed that the magnetic flux carried by the downflows leaves the box unimpeded. In order to always ensure that the diffusive boundary layer is well resolved numerically and to reduce the effect of the numerical boundary on the magnetic fields in the bulk of the simulation domain, the magnetic diffusivity is increased in a region of $150 \mathrm{~km}$ thickness at the bottom boundary. The lower boundary condition also prevents the advection of horizontal magnetic flux into the box from below. In the real Sun, of course, magnetic flux from the deeper layers is probably advected into the surface layers and may influence the properties of the QS magnetic field. In our experiment, we intentionally exclude this source of magnetic flux in order to study local dynamo action in isolation.

While we have a constant value of the magnetic diffusivity in the box (outside the region of enhanced diffusivity near the bottom), the code uses an artificial viscosity that varies in space and time (see Vögler et al. 2005). Consequently, we can evaluate the magnetic Reynolds number, $R_{\mathrm{m}}$, for a simulation run, but a quantitative estimate of the hydrodynamic Reynolds number, $\mathrm{Re}$, and of the magnetic Prandtl number, $\operatorname{Pr}_{\mathrm{m}}=R_{\mathrm{m}} / \mathrm{Re}$, is difficult to obtain. Since both, the explicit magnetic diffusivity and the artificial viscosity, lead to a diffusive cutoff at the scale of the grid resolution, $\mathrm{Re}$ and $R_{\mathrm{m}}$ are of the same order of magnitude and thus $\operatorname{Pr}_{\mathrm{m}}$ is about unity at the smallest resolved spatial scales. For smooth flows on larger scales, the artificial viscosity is much smaller than the magnetic diffusivity, so that our "effective" magnetic Prandtl number on these scales is smaller than unity. Given these conditions, our results do not provide a proper basis for commenting on the much debated question of the dependence of turbulent dynamo action on the value of $\operatorname{Pr}_{\mathrm{m}}$ (e.g., Boldyrev \& Cattaneo 2004; Ponty et al. 2005; Schekochihin et al. 2005; Brandenburg \& Subramanian 2005).

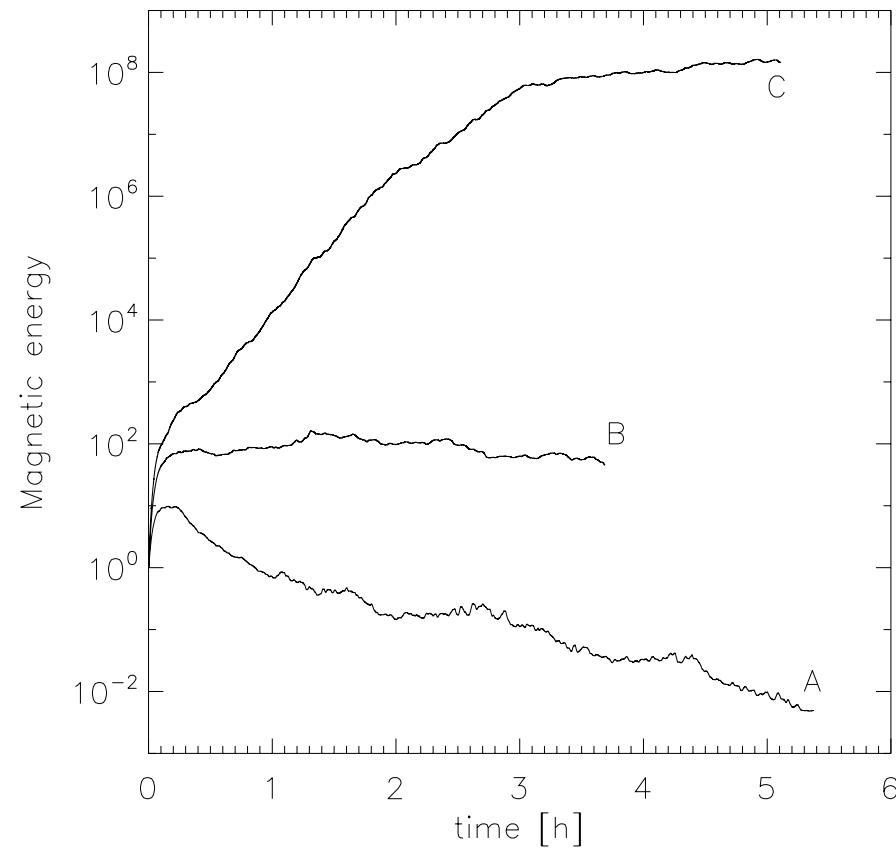

Fig. 1. Total magnetic energy (normalized to the energy of the initial seed field at $t=0$ ) in the simulation box as a function of simulated solar time for the three runs specified in Table 1. All runs show an initial rapid energy increase due to flux expulsion of the seed field by granulation. Thereafter, run A shows an exponential decay with an $e$-folding time of roughly one hour, while run $\mathrm{B}$ is approximately marginal at a low energy level. Run $\mathrm{C}$ exhibits exponential growth with a time scale of about 10 minutes and approaches a saturation level of a few percent of the total kinetic energy of the convective flow.

We have carried out three simulation runs with different magnetic Reynolds numbers, $R_{\mathrm{m}}$. For all runs, a weak magnetic seed field with zero net flux was introduced into a fully developed nonmagnetic convection pattern. The seed field was purely vertical, with polarity variations corresponding to a checkerboard-like $4 \times 4$ horizontal planform and a constant field strength of $\left|B_{0}\right|=10 \mathrm{mG}$. The box dimensions, grid resolution, and magnetic diffusivities for the runs are given in Table 1.

\section{Results}

Figure 1 shows the magnetic energy for the three simulation runs as a function of time. In run $\mathrm{A}\left(R_{\mathrm{m}} \approx 300\right)$, the magnetic energy decays exponentially after a brief initial amplification due to flux expulsion acting on the initial magnetic configuration. Run B $\left(R_{\mathrm{m}} \approx 1300\right)$ appears to be close to the point of marginal dynamo excitation. The magnetic energy levels out well within the kinematic regime, with maximum field strengths several orders of magnitude below local equipartition with respect to the kinetic energy density of the flow. Finally, run C $\left(R_{\mathrm{m}} \approx 2600\right)$ exhibits exponential growth with an $e$-folding timescale of roughly 10 minutes. After about three hours of simulated solar time, the magnetic energy approaches a saturated level of about $3 \%$ of the total kinetic energy of the convective flow.

For a snapshot during the saturation phase of run C, Fig. 2 shows maps of the (bolometric) brightness and of the vertical magnetic field on two surfaces of constant (Rosseland) optical depth, $\tau_{\mathrm{R}}$. Around $\tau_{\mathrm{R}}=1$, the field exhibits an intricate smallscale mixed-polarity structure, which extends down to the diffusive length scale. The (unsigned) mean magnetic flux density at this level has reached a value of about $25 \mathrm{G}$. At the 

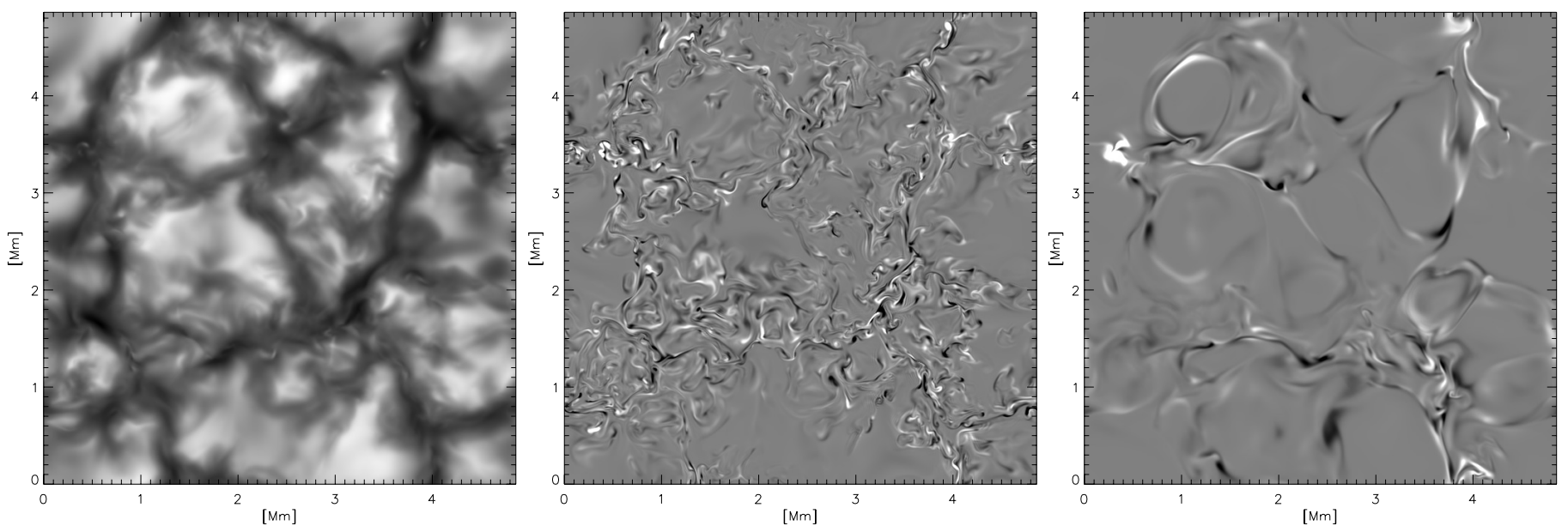

Fig. 2. Snapshot from the dynamo run $\mathrm{C}$, taken about $5 \mathrm{~h}$ after introducing the seed field. The vertically emerging bolometric intensity (brightness, left panel) reveals a normal solar granulation pattern. The other panels show the vertical component of the magnetic field on two surfaces of constant (Rosseland) optical depth, $\tau_{\mathrm{R}}$. Near the visible surface (middle panel, $\tau_{\mathrm{R}}=1$, grey scale saturating at $\pm 250 \mathrm{G}$ ), the magnetic field shows an intricate small-scale pattern with rapid polarity changes and an unsigned average flux density of $25.1 \mathrm{G}$. About $300 \mathrm{~km}$ higher, at the surface $\tau_{\mathrm{R}}=0.01$ (right panel, grey scale saturating at $\pm 50 \mathrm{G}$ ), the unsigned average flux density has decreased to $3.2 \mathrm{G}$ and the field distribution has become considerably smoother, roughly outlining the network of intergranular downflow lanes (darker areas on the left panel).

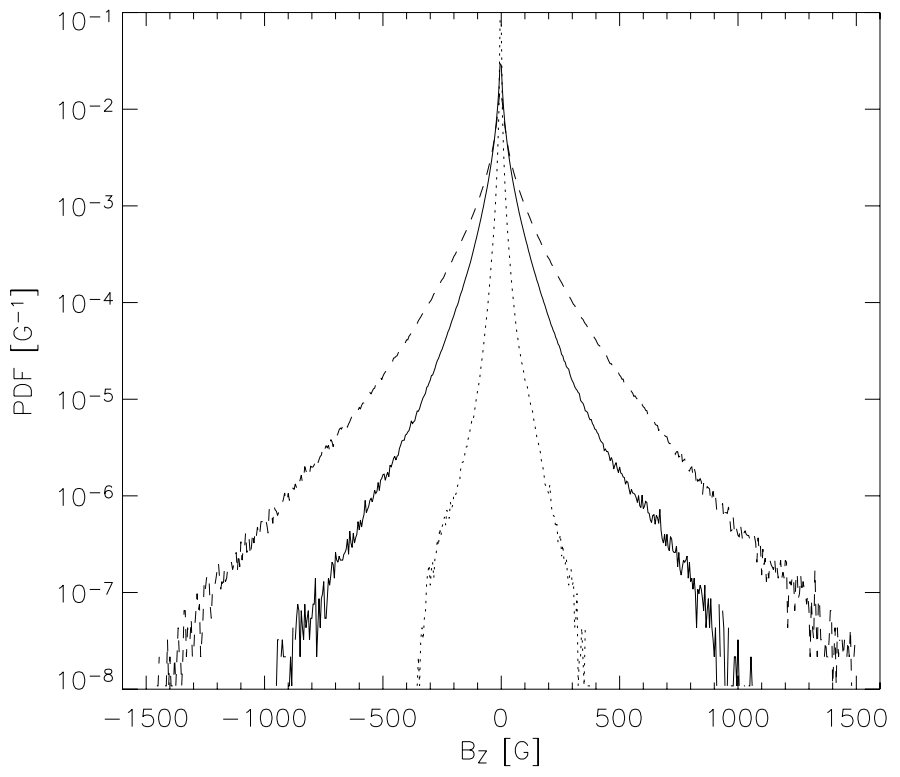

Fig. 3. Probability density function (PDF) for the vertical field component at three different geometrical height levels. Dashed curve: $z=$ $-370 \mathrm{~km}$ (about $450 \mathrm{~km}$ below the visible surface); solid curve: $z=$ $80 \mathrm{~km}$ (roughly corresponding to the average level of $\tau_{\mathrm{R}}=1$ ); dotted curve: $z=400 \mathrm{~km}$ (about the average level of $\tau_{\mathrm{R}}=0.01$ ). Shown are time averages over about 20 minutes around $t \simeq 4.5 \mathrm{~h}$.

surface $\tau_{\mathrm{R}}=0.01$, about $300 \mathrm{~km}$ higher in the atmosphere, the spatial distribution of the field is significantly smoother and more closely associated to the intergranular downflow lanes. The mean flux density has decreased to about $3 \mathrm{G}$. This strong decrease indicates the absence of significant dynamo driving in the convectively stable layers above $\tau_{\mathrm{R}}=1$, so that the field decays rapidly with height, owing to its small horizontal spatial scale near the visible solar surface.

Figure 3 shows the average probability density function (PDF) of the vertical magnetic field, determined during the saturation phase of the dynamo, at three height levels. The PDFs have the form of stretched exponentials, indicating a strong intermittency of the magnetic field at all heights. The strongest magnetic features occasionally reach vertical field strengths beyond $1 \mathrm{kG}$ near $\tau_{\mathrm{R}}=1$.

Energy spectra for the vertical components of the nearsurface magnetic field and velocity as a function of horizontal wave number, $k_{\mathrm{h}}$, are given in Fig. 4 . The spectral magnetic energy shows a broad peak at $k_{\mathrm{h}} \simeq 30$, which corresponds to a wavelength of about $200 \mathrm{~km}$. At the high-wavenumber end of the spectra, the magnetic and kinetic energies become less disparate. The remaining deviation from equipartition is due to the anisotropy resulting from the strong stratification.

We find that convective downward pumping of flux in fact has a significant effect on the energy balance of the dynamo, as conjectured by Stein \& Nordlund (2003). At any given height, the time dependence of the horizontally averaged magnetic energy density, $e_{\mathrm{mag}}$, is governed by the equation $\partial_{\mathrm{t}} e_{\mathrm{mag}}=W_{\mathrm{L}}-$ $W_{\mathrm{J}}-\partial \mathcal{P}_{z} / \partial z . W_{\mathrm{L}}$ is the rate of work against the Lorentz force, $W_{\mathrm{J}}$ is the Joule heating rate, and $\mathcal{P}_{z}$ is the vertical component of the Poynting flux, the advective part of which measures the draining of magnetic energy due to convective pumping. All quantities are meant to be horizontal averages. The advective Poynting flux is negative throughout the convectively unstable parts of the simulation domain, confirming that any growth of magnetic energy in the system must have its source inside the domain. The diffusive part of $\mathcal{P}_{z}$ is found to be negligible in the convecting layer. In the absence of convective pumping, the difference $W_{\mathrm{L}}-W_{\mathrm{J}}$ would be a measure for the growth of the magnetic energy during the exponential growth phase. In our case, more than $80 \%$ of this difference is indeed carried downwards by means of the term $-\partial \mathcal{P}_{z} / \partial z$ and leaves the box through the bottom boundary. However, the effect only reduces the growth rate but does not shut down dynamo action if the magnetic Reynolds number is sufficiently large.

\section{Discussion}

Our main finding is that a realistic flow topology of strongly stratified convection in the near-surface layers of the Sun is capable of sustaining dynamo action. Downward pumping in an open box has a significant impact on the energy balance, but is not able 


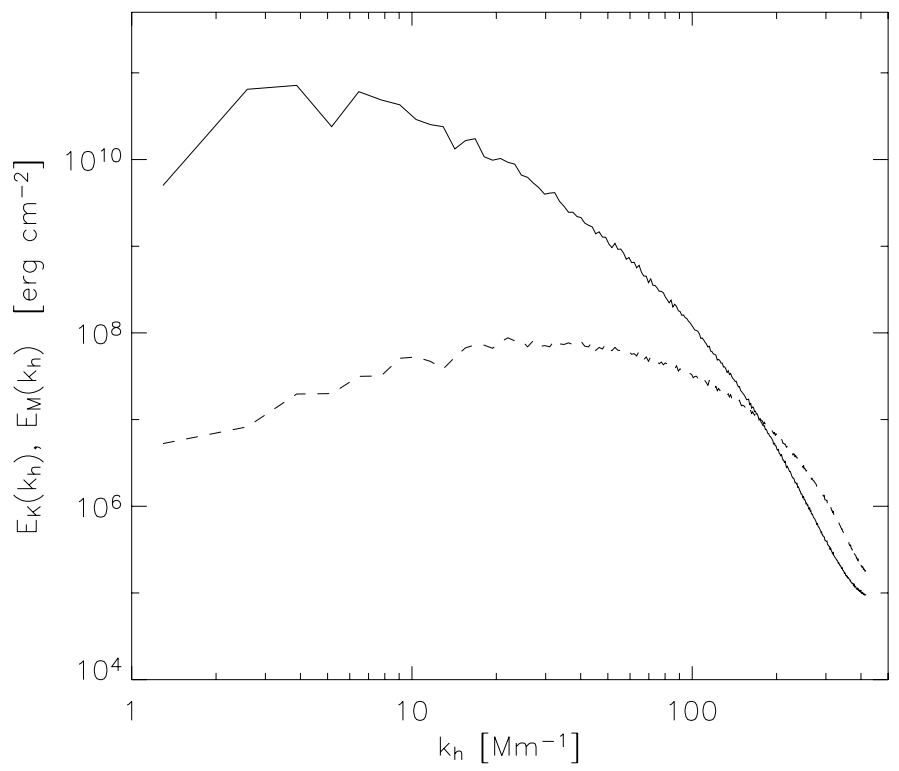

Fig. 4. Energy spectra based on the vertical components of velocity and magnetic field, respectively, as functions of horizontal wave number, $k_{\mathrm{h}}$. The values are taken at $z=0$, corresponding to a depth of about $80 \mathrm{~km}$ below the average average level of $\tau_{\mathrm{R}}=1$ ), at $t \simeq 5 \mathrm{~h}$. The kinetic energy spectrum (solid curve) peaks at $k_{\mathrm{h}} \simeq 3 \ldots 4 \mathrm{Mm}^{-1}$, roughly corresponding to the typical scale of granules. The magnetic energy spectrum has a broad maximum around wave numbers of about $30 \mathrm{Mm}^{-1}$, corresponding to length scales of at least an order of magnitude smaller.

to shut down the dynamo, presumably because there is sufficient local recirculation (e.g. by turbulent entrainment of downflowing material into upflow regions) to amplify the magnetic field near the surface. A more detailed analysis will have to be carried out in order to clarify the physical mechanism at work here. Strictly speaking, a dynamo simulation would have to be run for several global diffusive timescales before transients can be definitively ruled out. This is practically not feasible in our case since the global diffusion time for run $\mathrm{C}$ is about 18 days. On the other hand, the exponential amplification of a miniscule seed field to substantial saturation levels of flux over several hours, with a growth time corresponding to the granulation time scale, lends credibility to physical relevance to the results.

While demonstrating the possibility of local dynamo action by granulation in principle, the quantitative results of our simulations should be interpreted with caution. Owing to our subgrid model for the viscosity, the magnetic Prandtl number cannot be uniquely defined. We have argued that its value is significantly smaller than unity at the spatial scales where the magnetic energy peaks, but certainly our numerical experiment is not suited to address the question of turbulent dynamo action in the limit of very small $\operatorname{Pr}_{\mathrm{m}}$. Likewise, the saturation level of our dynamo run probably depends on the magnetic Reynolds number (as well as on $\operatorname{Pr}_{m}$ ), so that a quantitative comparison with observational results in terms of mean flux densities and PDFs would certainly be premature. Such comparison would also have to take into account, in addition to the self-excited dynamo, the advection of flux from below and the processing of magnetic debris from decaying active regions. Still, we deem it noteworthy that levels of magnetic flux of the observed order of magnitude can, in principle, be produced by simulations of the kind presented here.

This is just a first step and much remains to be done in order to establish quantitatively reliable results of near-surface local dynamo action. This includes studying the effects of deeper and wider computational boxes as well variations of the boundary conditions. Clearly, the dependence of the saturation level on the magnetic Reynolds and magnetic Prandtl numbers has to be investigated through series of controlled numerical experiments. Comparison with observational results will be crucial in order to evaluate the contributions by flux advection from the deeper layers.

Acknowledgements. We are grateful to Robert Cameron and Matthias Rempel for stimulating and helpful discussions.

\section{References}

Boldyrev, S., \& Cattaneo, F. 2004, Phys. Rev. Lett., 92, 144501 Brandenburg, A., \& Subramanian, K. 2005, Phys. Rep., 417, 1 Cattaneo, F. 1999, ApJ, 515, L39

Cattaneo, F., Emonet, T., \& Weiss, N. 2003, ApJ, 588, 1183

Domínguez Cerdeña, I., Kneer, F., \& Sánchez Almeida, J. 2003, ApJ, 582, L55 Domínguez Cerdeña, I., Almeida, J. S., \& Kneer, F. 2006a, ApJ, 646, 1421 Domínguez Cerdeña, I., Sánchez Almeida, J., \& Kneer, F. 2006b, ApJ, 636, 496 Hagenaar, H. J., Schrijver, C. J., \& Title, A. M. 2003, ApJ, 584, 1107

Khomenko, E. V., Collados, M., Solanki, S. K., Lagg, A., \& Trujillo Bueno, J. 2003, A\&A, 408, 1115

Lin, H., \& Rimmele, T. 1999, ApJ, 514, 448

Lites, B. W., \& Socas-Navarro, H. 2004, ApJ, 613, 600

Petrovay, K., \& Szakaly, G. 1993, A\&A, 274, 543

Ponty, Y., Mininni, P. D., Montgomery, D. C., et al. 2005, Phys. Rev. Lett., 94, 164502

Sánchez Almeida, J. 2003, A\&A, 411, 615

Sánchez Almeida, J. 2005, A\&A, 438, 727

Sánchez Almeida, J., \& Lites, B. W. 2000, ApJ, 532, 1215

Sánchez Almeida, J., Domínguez Cerdeña, I., \& Kneer, F. 2003, ApJ, 597, L177

Schekochihin, A. A., Haugen, N. E. L., Brandenburg, A., et al. 2005, ApJ, 625, L115

Stein, R. F., \& Nordlund, A. 1989, ApJ, 342, L95

Stein, R. F., \& Nordlund, A. 2003, in Modelling of Stellar Atmospheres, ed. N. Piskunov, W. W. Weiss, \& D. F. Gray (San Francisco, California: ASP), 169, IAU Symp., 210

Trujillo Bueno, J., Shchukina, N., \& Asensio Ramos, A. 2004, Nature, 430, 326 Vögler, A. 2003, Ph.D. Thesis, University of Göttingen, Germany, http://webdoc.sub.gwdg.de/diss/2004/voegler

Vögler, A., Shelyag, S., Schüssler, M., et al. 2005, A\&A, 429, 335 